\title{
Could Theophylline Be First-Line Treatment for Post-Spinal Puncture Headache in Postpartum Mothers?
}

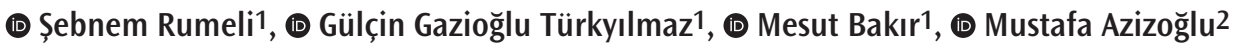 \\ 1Department of Algology, Mersin University School of Medicine, Mersin, Turkey \\ 2Department of Anesthesiology and Reanimation, Mersin University School of Medicine, Mersin, Turkey
}

\section{Abstract}

BACKGROUND/AIMS: Theophylline is a drug proven safe to use during the lactation period. This study retrospectively evaluates the efficacy of theophylline infusion administered under a standard protocol in the treatment of post-spinal puncture headache (PSPH) following a cesarean delivery, and discusses its role in the treatment measures in regard to its effects on the postpartum mother and infant.

MATERIALS and METHODS: A total of 33 patients who underwent a designated treatment algorithm at our Algology department were included in the study. Information on patient age, level of spinal block administration, and needle diameter were recorded from the patient records. Examination findings, analgesic drugs used before the hospital admission, onset of headache following a spinal block, time from the onset until theophylline administration, number of theophylline infusions, and Visual Analogue Scale (VAS) scores before and after the treatment were questioned in headache evaluation. Regarding drug safety, changes in the blood pressure and heart rate before and after the drug administration were evaluated.

RESULTS: Seventeen (51.5\%) patients did not receive the additional drug treatment before our evaluation, and only received bed rest and fluid therapy. Mean standing VAS score was $6.1 \pm 1.8$ before a theophylline infusion and $1.3 \pm 1.245$ mins after the end of the infusion ( $p<0.05$ ). Infusion was administered once in 22 (66.7\%) of the patients. Headache had resolved in all the 31 (93.9\%) patients who arrived for an evaluation 24 and/or 48 hours after the theophylline administration.

CONCLUSION: Theophylline infusion provided a rapid and effective treatment of PSPH in postpartum mothers. Despite the limitations, we believe our study contains valuable findings that may be helpful in studies on planning a "PSPH treatment algorithm" for postpartum mothers.

Keywords: Post-spinal puncture headache; theophylline; postpartum; mother; infant

\section{INTRODUCTION}

Post-spinal puncture headache (PSPH) is one of the most common complications of spinal anesthesia. Pregnant women have several risk factors associated with the PSPH development due to the gender and age distribution. ${ }^{1}$ While its incidence following a spinal anesthesia is reported between $3 \%-10 \%$, this number may rise to $32 \%$ after a cesarean delivery. ${ }^{1,2}$
There are serious debates on the effectiveness of the bed rest and fluid therapy as conservative treatment options of PSPH. Among the drug options, only caffeine, theophylline, gabapentin, and hydrocortisone have been determined to be effective, ${ }^{3}$ in which only caffeine and theophylline have been listed as safe, to use during the lactation period. ${ }^{4}$ There are also studies indicating that theophylline infusion could be used for PSPH prophylaxis following a cesarean delivery. ${ }^{5}$

To cite this article: Rumeli ș, Gazioğlu Türkyılmaz G, Bakır M, Azizoğlu M. Could Theophylline Be First-Line Treatment For Post-Spinal Puncture Headache in Postpartum Mothers. Cyprus J Med Sci 2021;6(Suppl 1):43-47

ORCID iDs of the authors: S.R. 0000-0002-7042-6934; G.G. 0000-0003-4148-1958; M.B. 0000-0002-3627-3882; M.A. 0000-0002-8266-5203.

Address for Correspondence: Șebnem Rumeli

E-mail: sebnemrumeli66@gmail.com

ORCID ID: orcid.org/0000-0002-7042-6934
Received: 03.01.2020

Accepted: 07.06.2020

Copyright 2021 by the Cyprus Turkish Medical Association / Cyprus Journal of Medical Sciences published by Galenos Publishing House.

Content of this journal is licensed under a Creative Commons Attribution 4.0 International License 
Pain while standing up is one of the most prominent features of PSPH diagnosis, and the reason patients avoid mobilization. Prolonged immobilization in the postpartum period increases the maternal risk of deep vein thrombosis and pulmonary embolism. Pain and limited mobility reduce the mother's daily activity and delay in caring for her infant. There is currently no treatment algorithm for PSPH which takes these events under a consideration.

Our department practices a standard protocol consisting of theophylline infusion for PSPH treatment. In this study, we aim to conduct a retrospective analysis of the patients who were administered with theophylline infusion to treat PSPH after a cesarean delivery to evaluate efficacy of the drug and discuss the order of the treatment steps for a lactating mother in context to the literature.

\section{MATERIALS AND METHODS}

After obtaining ethics committee approval (date: 10.05.2018; decision: 2018/202), the follow-up and treatment files of 33 patients who underwent the treatment algorithm at our Algology department due to PSPH between 2009-2018 were examined. Since our study was retrospective, informed consent was not obtained. Information on patient age as well as additional diseases, spinal block injection level, and the needle diameter were recorded. Examination findings, analgesic drugs used before the hospital admission, onset of headache following a spinal block, time from onset until the theophylline infusion, number of theophylline applications, and Visual Analogue Scale (VAS) scores before and after the treatment were questioned in headache evaluation. Regarding the drug safety, changes in the blood pressure and heart rate before and after the drug administration were also recorded.

\section{PSPH Evaluation and Treatment Algorithm}

According to the algorithm, patients diagnosed with PSPH with VAS of $<4$ were recommended with a conservative treatment. Neurological examination was repeated 24 and/or 48 hours with a follow-up when deemed necessary by the doctor. Patients with VAS $\geq 4$ at follow-up examination or admission were administered with $200 \mathrm{mg}$ IV theophylline as $45 \mathrm{~min}$ infusion with a hemodynamic monitorization. Forty-five minutes after the infusion, patients were asked to sit, and VAS scores were reassessed. Patients whose symptoms regressed or were able to be mobilized were discharged and called for follow-up 24 and/ or 48 hours later after the discharge. Patients decided to have a repeated theophylline infusion were hospitalized and closely monitored. Patients with change in type of headache, increased severity, presence of pathological findings in neurological examination (such as cranial nerve examination pathology, motor-sensory deficit, visual field examination), history of cerebrovascular accident, and previous intracranial surgery underwent cranial and/or spinal magnetic resonance imaging (MRI). Patients with the intracranial hypotension findings in cranial MRI and VAS $\geq 4$ despite passing of 48 hours were planned with an epidural patch. Theophylline may be re-administered to reduce the symptoms until blood patch procedure.

Unlike the other patients, the lactating mother is informed that breastfeeding must be postponed two hours after a theophylline infusion.

\section{Statistical Analysis}

For statistical analysis, data was input into "Statistical Package for the Social Sciences" version 24 (SPSS v.24) SPSS Inc., Chicago, IL, USA) and "e-PICOS" program was used for calculations based on "Medicres Good Biostatistical Practices." Descriptive statistics were used for the categorical variables and frequency calculations were expressed as percentage. Comparisons of means were performed with independent t-test. $P$-value $<0.05$ was considered as statistically significant.

\section{RESULTS}

Mean patient age was $31.6 \pm 4.6$ years. Of the PSPH patients, $32(96.7 \%)$ were evaluated at the gynecology and obstetrics department, and one (3.3\%) with neurology consultation.

Lumbar puncture was performed at $\mathrm{L}_{3}-\mathrm{L}_{4}$ level in 17 (51.5\%) patients and $L_{4}-L_{5}$ in nine (27.3\%) patients. Seven (21.2\%) patients did not have the available records on lumbar puncture level.

$22 \mathrm{G}$ needle was used for lumbar puncture in 27 (81.8\%) patients. Information on needle diameter was unavailable in six (18.2\%) patients.

The number of patients with orthostatic headache in examination was $21(63.6 \%)$. Twelve (36.3\%) patients had more than two additional complaints (Table 1). There were no complaints of fever or neck stiffness.

\begin{tabular}{|l|l|l|}
\begin{tabular}{|l|l|} 
Table 1. Additional complaints in patients with an \\
orthostatic hypotension
\end{tabular} & Number & Percentage (\%) \\
\hline Complaint & 6 & 18.18 \\
\hline Nausea & 6 & 18.18 \\
\hline Tinnitus & 4 & 12.12 \\
\hline Dizziness & 2 & 6.06 \\
\hline Vomiting & 2 & 6.06 \\
\hline Diplopia & 3 & 9.09 \\
\hline Photophobia & 3 & 9.09 \\
\hline Phonophobia & 2 & 6.06 \\
\hline Numbness in left arm & 1 & 3.03 \\
\hline Prickling/tingling & 1 & 3.03 \\
\hline Ear fullness & &
\end{tabular}


Onset of headache was $<23 \mathrm{~h}$ in 21 (67.7\%) patients and 24-120 h in 12 (32.3\%) patients. Before our evaluation, a total of 17 patients (51.5\%) did not receive any additional drug treatment, but they were only on bed rest and fluid intake. Eleven (33.3\%) patients received paracetamol (500 mg)-codeine (10 mg) combination; five patients (15.2\%) received paracetamol (300-500 mg) treatment.

Mean time from headache onset until theophylline administration was 85.2 254.2 h (min: 24/h; max: 264/h). Theophylline infusion was initiated $24-120 / \mathrm{h}$ after the onset in 27 patients (81.4\%) and $>121$ hours in five (15.6\%) patients. Time record of theophylline administration was unavailable in one patient.

VAS score was $\geq 4$ in all the patients (100\%). Mean standing VAS score was $6.1 \pm 1.8$ before theophylline the infusion, and $1.3 \pm 1.2$ 45 minutes after ending the infusion ( $p=0.001$ ) (Figure 1).

Patients with complaints in addition to orthostatic headache did not have a significant difference in mean VAS score before and after the theophylline infusion compared to those who did not (6.10 $\pm 1.2,6.16 \pm 2.1 ; 0.8 \pm 1.3,1.6 \pm 1.1$, respectively; $p>0.05$ all).

Mean heart rate was $74.8 \pm 10.4 \mathrm{bpm}$ before theophylline infusion and 78.0 $\pm 11.1 \mathrm{bpm}$ after the infusion $(p<0.05)$. Mean diastolic blood pressure was $73.1 \pm 9.6 \mathrm{mmHg}$ before the infusion and $71.4 \pm 6.4 \mathrm{mmHg}$ after the infusion $(p>0.05)$.

Theophylline infusion was administered only once in 22 (66.7\%) patients. As for the remaining patients, theophylline was administered twice in seven (21.2\%) and three times in four (12.1\%) patients. Two of the patients who were administered theophylline three times were decided to receive an epidural

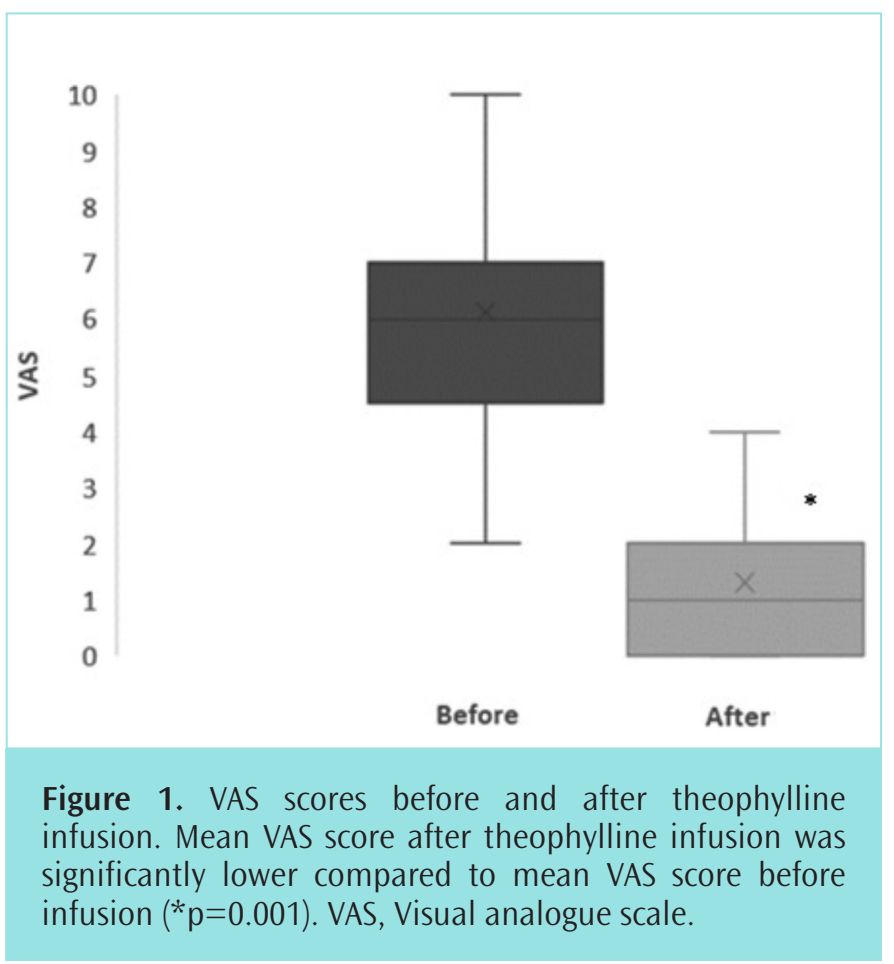

blood patch. During the time until the patch procedure, infusions were repeated to reduce thesymptoms.

Adverse effects associated with the theophylline were not reported in any of the records.

Headache had resolved in all the 31 patients (93.9\%) who arrived for follow-up evaluation 24 and/or 48 hours after the theophylline infusion. In the two patients who received blood patch (6.1\%), headache had resolved but diplopia continued. Records showed that ophthalmologic consultation was requested for these patients for follow-up and treatment.

\section{DISCUSSION}

This study was the first retrospective study on theophylline administration for PSPH treatment in the postpartum period within the framework of a designated algorithm. Success rate with a single theophylline infusion was $66.7 \%$. This rate increased to $87 \%$ after the second infusion. While VAS scores of all the patients were "severe pain" level at admission, they improved to "no pain" level after the infusion. We believe this change is significant, especially regarding mothers taking care of themselves and their infants in as short period as possible.

According to The International Classification of Headache Disorders (ICHD)-3 beta version, PSPH is defined as an orthostatic headache that occurs within five days of a Dural puncture. ${ }^{6}$ All patients in our study had headache that developed within five days after the Dural puncture. Theophylline administration time varied between 1-11 days.

There are some critical studies on the use of the drugs that are effective in the treatment of PSPH. Caffeine has been shown to provide temporary relief but requires repeated doses and does not reduce the need for epidural blood patch., ${ }^{3,7}$ Although the efficacy of the hydrocortisone has been shown, the authors emphasize that the alternatives with a higher efficacy and broader presence in the literature should be prioritized., 3-9 Gabapentin is a moderately safe drug option during the lactation period. However, there is a reluctance to use the drug with the newborns due to concerns that it may cause neonatal neurotoxicity caused by slow drug clearance. ${ }^{10,11}$ Although the use of NSAIDs, acetaminophen, and oxycodone, which are also among the drug treatments of $\mathrm{PSPH}$, are safe during the lactation period, their efficacy in PSPH treatment has been found to be low. ${ }^{7,8}$ In our patients, $48.5 \%$ had received a conservative drug therapy which they did not benefit from.

There are studies that demonstrate the efficacy of theophylline in the PSPH treatment. Ergun et al. ${ }^{12}$ conducted a study on 20 patients and reported a significant improvement in the VAS scores, 30 minutes after the administration in $47.1 \%$ and 60 minutes after in $61.9 \%$ of the patients. Another study on 35 patients which compared theophylline to a placebo found 
that the group which received only $200 \mathrm{mg}$ theophylline showed a significant improvement in the VAS scores after 40 minutes..$^{13}$ In the same study, patients with $<50 \%$ decrease in the VAS score received the repeated infusion of theophylline at the same dose and time and significantly improved. ${ }^{13}$ In our study, $66.7 \%(\mathrm{n}=22)$ of patients had significantly less pain after the first infusion. The remaining seven patients (21\%) did not require any additional treatment after the second infusion.

There are also studies that associate needle diameter of the puncture and severity of pain in PSPH. It has been indicated that the use of needles with larger diameter cause a larger lesion and more CSF leakage in the dura mater. ${ }^{14} 22 \mathrm{G}$ spinal needles had been used in $81.8 \%$ of our patients. Even in these $22 \mathrm{G}$ needle blocks, defined as a large diameter, theophylline treatment reduced the other neurological symptoms in addition to VAS values, and we believe this may be considered as another finding indicating the efficacy of the drug.

Theophylline requires monitorization during the administration due to its cardiac effects. Andreas et al. ${ }^{15}$ reported in 2004 that intravenous theophylline infusion raised systolic blood pressure and heart rate in the congestive heart failure patients, but this was not statistically significant. In our study, there was no statistically significant difference in systolic and diastolic blood pressure before and after the theophylline infusion. Although there was a statistically significant difference in the heart rate, it was not clinically significant. One study that investigated the kinetics of maternal theophylline transfer to breast milk reported that $<1 \%$ of maternal theophylline dose transferred into a breast milk. In the same study, theophylline peak breast milk levels manifested immediately after the IV administration and decreased over a time, indicating that the amount of theophylline consumed by the infants would depend on the volume of breast milk and the concurrent plasma concentration of the mother. ${ }^{16}$ In another study, no adverse effects were observed in four out of five breastfed threeday-old infants who were administered $200 \mathrm{mg}$ intravenous aminophylline treatment every six hours for an asthma treatment, and had no time limitation for breastfeeding. Irritability and sleep disturbance were observed in one infant and the symptoms completely disappeared when the drug treatment was discontinued..$^{17}$ The main reason breastfeeding time was specified as two hours from the theophylline infusion was that we believe the drug concentration will decrease considerably in this period. ${ }^{18}$

\section{Limitations of the Study}

While our study was conducted according to the protocol, there were also some limitations. Limited mobilization was not evaluated with a standardized score, theophylline levels were not assessed in infants, and the drug was not followed up for adverse effects, preventing more concise findings. We believe prospective studies may consider these points in the future.

\section{CONCLUSION}

In conclusion, theophylline infusion provided rapid and effective treatment of PSPH in postpartum mothers. We believe our study contains valuable information that may be helpful in prospective studies planning a "PSPH treatment algorithm" in postpartum mothers.

\section{ETHICS}

Ethics Committee Approval: This study was approved by the Ethics Committee of Mersin University (date: 10.05.2018; decision no: 2018/202).

Informed Consent: Since this study was retrospective, informed consent was not obtained.

Peer-review: Externally peer-reviewed.

\section{Authorship Contributions}

Conception: \$.R., M.A.; Design: \$.R., G.G.T.; Supervision: \$̧.R., M.B.; Data Collection and/or Processing: G.G.T.; Analysis and/ or Interpretation: S.R., G.G.T.; Literature Review: M.B., M.A.; Writing: G.G.T., M.B., M.A.; Critical Review: \$.R., G.G.T., M.B., M.A.

\section{DISCLOSURES}

Financial Disclosure: The author declared that this study had received no financial support.

Conflict of Interest: The authors declare no conflict of interest.

\section{REFERENCES}

1. Ali HM, Mohamed MY, Ahmed YM. Postdural puncture headache after spinal anesthesia in cesarean section: Experience in six months in 2736 patients in Kasr El aini teaching hospital - Cairo University. Egypt J Anaesth. 2014;30:383-386.

2. Pirbudak L, Ozcan HI, Tumturk P. Postdural puncture headache: Incidence and predisposing factors in a university hospital. Agri. 2019;31:1-8.

3. Alam MR, Rahman MA, Ershad R. Role of very short-term intravenous hydrocortisone in reducing postdural puncture headache. J Anaesthesiol Clin Pharmacol. 2012;28:190-193.

4. Tetik BK TI. Drug use in breastfeeding mothers. TJFMPC. 2017;8:83-89.

5. Yang CJ, Chen T, Ni X, et al. Effect of pre-administration with aminophylline on the occurrence of post-dural puncture headache in women undergoing caesarean section by combined spinal-epidural anaesthesia. Int J Med Res. 2019;47:420-426.

6. Headache Classification Committee of the International Headache Society (IHS) The International Classification of Headache Disorders, 3rd edition. Cephalalgia. 2018;38:1-211.

7. Basurto Ona X, Osorio D, Bonfill Cosp X. Drug therapy for treating postdural puncture headache. Cochrane Database Syst Rev. 2015:CD007887. doi: 10.1002/14651858.CD007887.pub3 
8. Sachs HC, Committee on Drugs. The transfer of drugs and therapeutics into human breast milk: an update on selected topics. Pediatrics. 2013;132:796809 .

9. Baysinger CL, Pope JE, Lockhart EM, et al. The management of accidental dural puncture and postdural puncture headache: a North American survey. J Clin Anesthesia. 2011;23:349-360.

10. Bozdemir H, Peköz MT. The follow-up of epileptic patients in the birth and postpartum periods. Epilepsi. 2018;24:44-50.

11. Wagner $Y$, Storr F, Cope S. Gabapentin in the treatment of post-dura puncture headache: a case series. Anaesth Intensive Care. 2012;40:714-718.

12. Ergun U, Unal-Artik HA, Inan LE, et al. Intravenous theophylline rapidly decreases post- lumbar puncture headaches. Acta Neurologica Belgica. 2016;116:337-339.

13. Ergun U, Say B, Ozer G, et al. Intravenous theophylline decreases post-dural puncture headaches. J Clin Neurosci. 2008;15(10):1102-4. DOI: 10.1016/j. jocn.2007.11.001.
14. MS Ö. Post-dural puncture headache and treatment. Med J SDU/SDÜ Tıp Fak Derg. 2018;25:98-107.

15. Andreas S, Reiter H, Luthje L, et al. Differential effects of theophylline on sympathetic excitation, hemodynamics, and breathing in congestive heart failure. Circulation. 2004;110:2157-2162.

16. Stec GP, Greenberger P, Ruo TI, et al. Kinetics of theophylline transfer to breast milk. Clin Pharmacol Therap. 1980;28:404-408.

17. Yurchak AM, Jusko WJ. Theophylline secretion into breast milk. Pediatrics. 1976;57:518-520.

18. Drugs and Lactation Database. Aminophylline. Available at: https://www. ncbi.nlm.nih.gov/books/NBK501922/ 